\title{
Strain Control at Two-Dimensional Oxide Interfaces Probed by Aberration- Corrected STEM-EELS
}

\author{
David J. Baek ${ }^{1}$, Di Lu², Yasuyuki Hikita ${ }^{3}$, Harold Y. Hwang ${ }^{2,3}$ and Lena F. Kourkoutis ${ }^{4,5}$ \\ 1. School of Electrical and Computer Engineering, Cornell University, Ithaca, NY, USA. \\ 2. Department of Applied Physics, Stanford University, Stanford, CA, USA. \\ 3. Stanford Institute for Materials and Energy Sciences, SLAC National Accelerator Laboratory, Menlo \\ Park, CA, USA. \\ 4. School of Applied and Engineering Physics, Cornell University, Ithaca, NY, USA. \\ 5. Kavli Institute at Cornell for Nanoscale Science, Cornell University, Ithaca, NY, USA.
}

Perovskite oxides display a wide spectrum of functional properties such as superconductivity, colossal magnetoresistance, and ferromagnetism that can potentially be utilized for electronic device applications. Advances in the atomic-scale synthesis of these materials have enabled the design of novel oxide heterostructures exhibiting properties that are not found in their bulk constituents. Strain, for example, imposed on the overlaying epitaxial oxide film by a carefully chosen substrate, can alter the material's properties. Here, we use an alternative approach to controlling the strain in $\mathrm{Nd}_{0.5} \mathrm{Sr}_{0.5} \mathrm{MnO}_{3}$ (NSMO) films by inserting a flexible perovskite-like buffer layer (TSDA: $\mathrm{Sr}_{3} \mathrm{Al}_{2} \mathrm{O}_{6}$ ) between the film and the $\mathrm{SrTiO}_{3}$ (100) (STO) substrate [1]. Atomic-resolution mapping of the structure and chemistry at the NSMO/TSDA/STO interface reveals regions with uniform and defect-free buffer layers separated by regions where the buffer layer is lacking and where strain in the film is partially relieved by the formation of dislocations. The 1-nm wide dislocation cores are Mn rich and show a reduced $\mathrm{Mn}$ valence.

Previous work showed that when NSMO films are grown directly on STO substrates, misfit dislocations appear at the interface due to the $2.84 \%$ lattice mismatch $\left(\mathrm{a}_{\mathrm{STO}}=3.905 \AA, \mathrm{a}_{\mathrm{NSMO}}=3.797 \AA\right.$ ). The average spacing between the dislocation cores was found to be $\sim 30 \mathrm{~nm}$ [2]. However, in this work, the flexible nature of the TSDA buffer layer allows strain relief and the average spacing between dislocations is greatly enhanced to $\sim 100 \mathrm{~nm}$. Aberration-corrected ADF STEM imaging and spectroscopic mapping reveal that the dislocation cores are only present in areas that lack the uniform growth of the TSDA layer and which therefore lack an alternative strain release mechanism. The elemental maps in Fig. 1 correspond to an area with uniform growth of TSDA and interestingly, we observed selective Nd diffusion into the TSDA layer which suggests that the open crystal structure of TSDA allows preferential diffusion into the cation sites. However, in Fig. 2, the ADF STEM image and the elemental maps illustrate the missing TSDA layer around the dislocation core. From the Mn and Ti maps, the dislocation core is observed as being occupied by excess Mn and the corresponding site being Tideficient. Previously, similar diffusion of cations from the film into the open structure of the dislocation core was reported [3]. When the $\mathrm{Mn}-\mathrm{L}_{2,3}$ edge is integrated parallel to the interface, at the dislocation core, the peaks shift to lower energies suggesting a reduced Mn valence state. When compared with Mn$\mathrm{L}_{2,3}$ reference spectra, the $\mathrm{Mn}$ in the dislocation core corresponds to $\mathrm{Mn}^{3+}$ while that in NSMO is $\mathrm{Mn}^{3.5+}$. The Mn valence change was further confirmed by performing multivariate curve resolution (MCR) to extract the two distinct Mn components as presented in Fig. 2. A map of the spatial extents of these two components is obtained by a non-least squares fit to the $\mathrm{Mn}-\mathrm{L}_{2,3}$ edge spectroscopic map using the reference spectra extracted by MCR.

[1] D Lu et al, MRS Spring (2014), K12.11. 
[2] C. -P Chang et al, Nat. Commun. 5 (2014), p. 1.

[3] M Arredondo et al, Adv. Mater. 22 (2010), p. 2430.

[4] This work was supported by the Cornell Center for Materials Research with funding from the NSF MRSEC program (DMR-1120296) and the Department of Energy, Office of Basic Energy Sciences, Division of Materials Sciences and Engineering, under contract DE-AC02-76SF00515.

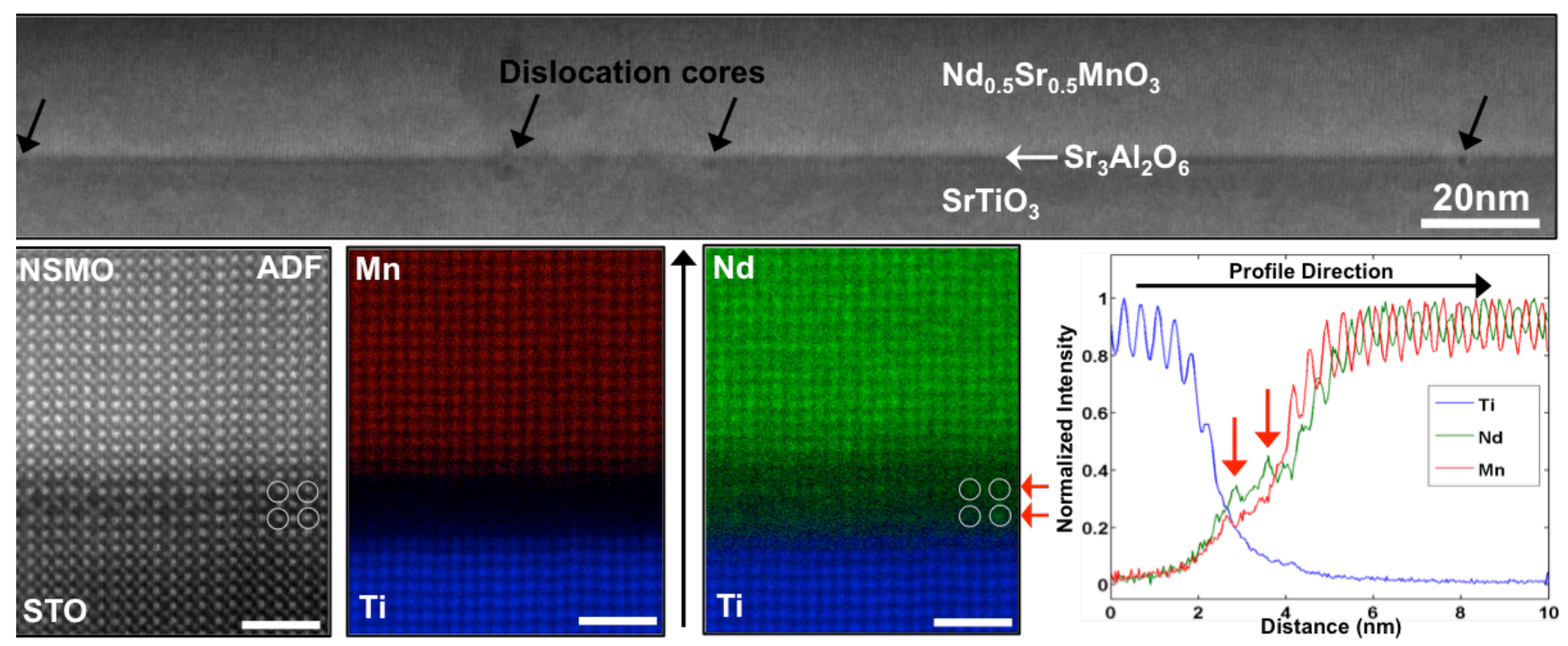

Figure 1. Upper figure: low magnification HAADF-STEM image showing the overall structure of the material under study. Lower figures from left to right: simultaneously recorded ADF image, elemental maps of $\mathrm{Ti}, \mathrm{Mn}$ and $\mathrm{Ti}, \mathrm{Nd}$ (white circles: selective diffusion of $\mathrm{Nd}$ ), concentration profile extracted from the elemental maps with red arrows indicating the location of $\mathrm{Nd}$ diffusion (scale bars: $2 \mathrm{~nm}$ ).
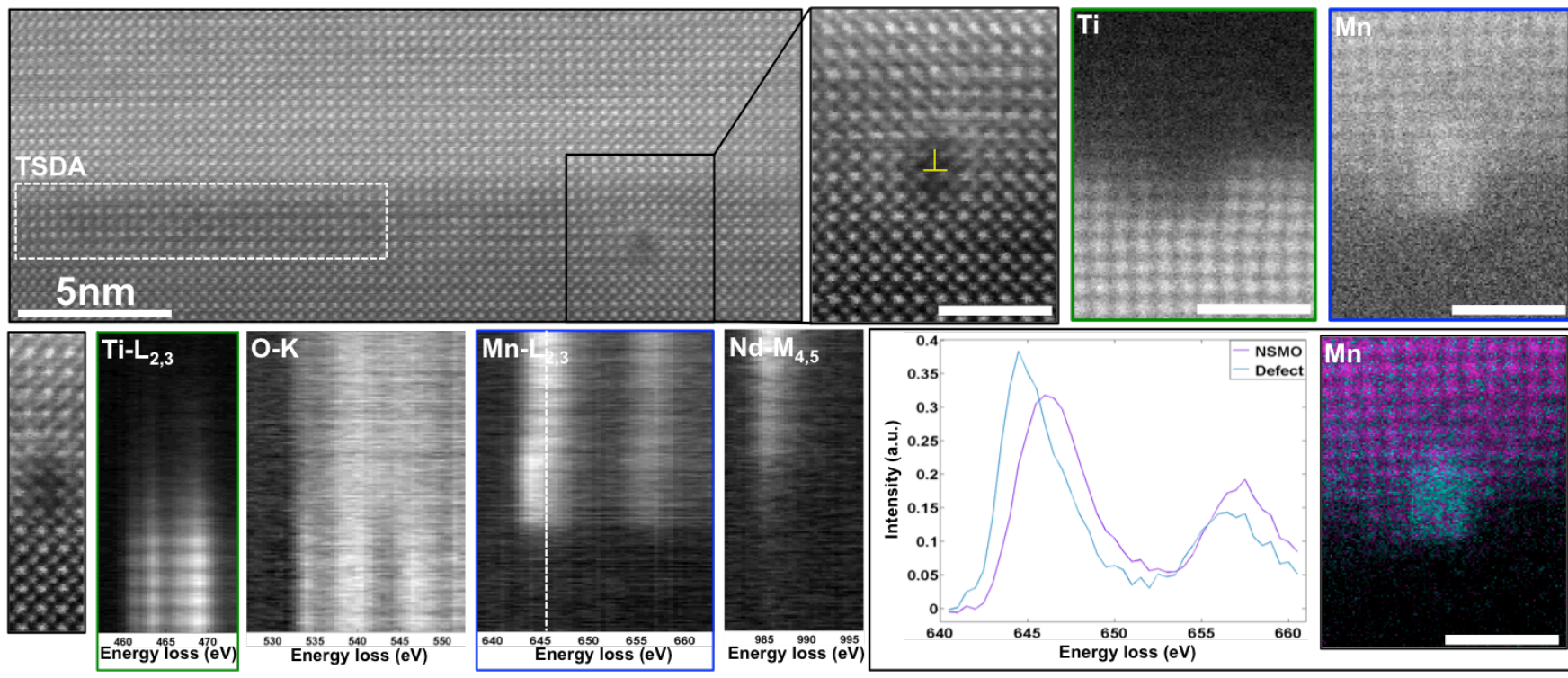

Figure 2. Upper figures: Closer look into the TSDA layer and the dislocation core, followed by elemental maps of $\mathrm{Ti}$ and $\mathrm{Mn}$ around the defect. Lower figures: Background-subtracted edge spectra with horizontal averaging (leftmost ADF indicates the area used for averaging). Rightmost mapping shows two distinct Mn components that are extracted by multivariate curve resolution. For the defect component, the major peaks of the $\mathrm{Mn}-\mathrm{L}_{2,3}$ edge shift to lower energies as shown in the left spectra (scale bars: $2 \mathrm{~nm}$ ). 